J. DIFFERENTIAL GEOMETRY

96 (2014) 201-222

\title{
INTERIOR CURVATURE ESTIMATES AND THE ASYMPTOTIC PLATEAU PROBLEM IN HYPERBOLIC SPACE
}

\author{
Bo Guan, Joel Spruck \& Ling Xiao
}

\begin{abstract}
We show that for a very general class of curvature functions defined in the positive cone, the problem of finding a complete strictly locally convex hypersurface in $\mathbb{H}^{n+1}$ satisfying $f(\kappa)=\sigma \in$ $(0,1)$ with a prescribed asymptotic boundary $\Gamma$ at infinity has at least one smooth solution with uniformly bounded hyperbolic principal curvatures. Moreover, if $\Gamma$ is (Euclidean) star-shaped, the solution is unique and also (Euclidean) star-shaped, while if $\Gamma$ is mean convex, the solution is unique. We also show via a strong duality theorem that analogous results hold in De Sitter space. A novel feature of our approach is a "global interior curvature estimate."
\end{abstract}

\section{Introduction}

Let $\mathbb{H}^{n+1}$ be the the hyperbolic space of dimension $n+1, n \geq 2$, and let $\partial_{\infty} \mathbb{H}^{n+1}$ denote the ideal boundary of $\mathbb{H}^{n+1}$ at infinity. In this paper we are concerned with the problem of finding complete hypersurfaces of constant curvature in $\mathbb{H}^{n+1}$ with prescribed asymptotic boundary at infinity. More precisely, given a disjoint collection of closed embedded smooth $(n-1)$-dimensional submanifolds $\Gamma=\left\{\Gamma_{1}, \ldots, \Gamma_{m}\right\} \subset \partial_{\infty} \mathbb{H}^{n+1}$, we seek a complete hypersurface $\Sigma$ in $\mathbb{H}^{n+1}$ satisfying

$$
f(\kappa[\Sigma])=\sigma
$$

with the asymptotic boundary

$$
\partial \Sigma=\Gamma
$$

where $f$ is a smooth symmetric function of $n$ variables, $\kappa[\Sigma]=\left(\kappa_{1}, \ldots, \kappa_{n}\right)$ denotes the induced hyperbolic principal curvatures of $\Sigma$, and $\sigma$ is a constant.

The problem was first studied by Anderson $[\mathbf{1}, \mathbf{2}]$, and Hardt and Lin [12] for area-minimizing varieties using geometric measure theory; their results were extended by Tonegawa $[\mathbf{2 0}]$ to hypersurfaces of constant mean curvature. In [15], Lin first used PDE methods to prove the

Received 12/17/2012. 
existence of smooth complete minimal hypersurfaces that are graphs in the upper half-space model over mean convex domains, followed by work of Nelli and Spruck [17] and Guan and Spruck [8] for hypersurfaces of constant mean curvature. For Gauss curvature, the asymptotic Plateau problem was initiated by Labourie $[\mathbf{1 4}]$ in $\mathbb{H}^{3}$ and by Rosenberg and Spruck $[\mathbf{1 8}]$ in $\mathbb{H}^{n+1}$. In recent work $[\mathbf{9}, \mathbf{1 0}, \mathbf{1 1}, \mathbf{1 9}]$ the authors considered the problem for more general curvature functions. In this paper we shall focus on locally strictly convex hypersurfaces and give a complete solution to problem (1.1)-(1.2) under very general assumptions on $f$.

Accordingly, we shall assume the curvature function $f$ to be defined on the positive cone $K_{n}^{+}:=\left\{\lambda \in \mathbb{R}^{n}\right.$ : each component $\left.\lambda_{i}>0\right\}$ with

$$
f=0 \text { on } \partial K_{n}^{+},
$$

and satisfy the fundamental structure conditions $[\mathbf{4}]$

$$
f_{i}(\lambda) \equiv \frac{\partial f(\lambda)}{\partial \lambda_{i}}>0 \text { in } K_{n}^{+}, \quad 1 \leq i \leq n,
$$

$f$ is a concave function in $K_{n}^{+}$.

Consequently,

$$
f>0 \text { in } K_{n}^{+} \text {. }
$$

For convenience we shall assume in addition that $f$ is normalized

$$
f(1, \ldots, 1)=1
$$

and is homogeneous of degree 1 :

$$
f(t \kappa)=t f(\kappa), \quad \forall t \geq 0, \kappa \in K_{n}^{+} .
$$

A hypersurface $\Sigma$ in $\mathbb{H}^{n+1}$ is said to be locally strictly convex if $\kappa[\Sigma] \in$ $K_{n}^{+}$; i.e., the principal curvatures of $\Sigma$ are positive everywhere.

In order to state our main results, it is convenient (without loss of any generality) to use the upper half-space model

$$
\mathbb{H}^{n+1}=\left\{\left(x, x_{n+1}\right) \in \mathbb{R}^{n+1}: x_{n+1}>0\right\}
$$

equipped with the hyperbolic metric

$$
d s^{2}=\frac{1}{x_{n+1}^{2}} \sum_{i=1}^{n+1} d x_{i}^{2} .
$$

Thus $\partial_{\infty} \mathbb{H}^{n+1}$ is naturally identified with $\mathbb{R}^{n}=\mathbb{R}^{n} \times\{0\} \subset \mathbb{R}^{n+1}$ and (1.2) may be understood in the Euclidean sense.

The first main result of this paper may be stated as follows. 
Theorem 1.1. Suppose $\Gamma=\partial \Omega \in C^{2}$ for a bounded domain $\Omega \subset$ $\mathbb{R}^{n}=\mathbb{R}^{n} \times\{0\}$ and $0<\sigma<1$. Under conditions (1.3)-(1.5) and (1.7)(1.8), there exists a complete locally strictly convex hypersurface $\Sigma$ in $\mathbb{H}^{n+1}$ satisfying (1.1)-(1.2) with uniformly bounded principal curvatures

$$
C^{-1} \leq \kappa_{i} \leq C \text { on } \Sigma \text {. }
$$

Moreover, $\Sigma$ is the vertical graph of $u \in C^{\infty}(\Omega) \cap C^{0,1}(\bar{\Omega}), u>0$ in $\Omega$, $u=0$ on $\partial \Omega, u^{2} \in C^{1,1}(\bar{\Omega})$, and

$$
u\left|D^{2} u\right|+|D u| \leq C \text { in } \bar{\Omega}, \sqrt{1+|D u|^{2}}=\frac{1}{\sigma} \text { on } \partial \Omega .
$$

Theorem 1.1 substantially improves our earlier results in $[\mathbf{9}, \mathbf{1 1}]$ where $\partial \Omega$ at least $C^{3}$ is needed as well as an additional more technical assumption

$$
\lim _{R \rightarrow+\infty} f\left(\lambda_{1}, \cdots, \lambda_{n-1}, \lambda_{n}+R\right) \geq 1+\varepsilon_{0} \quad \text { uniformly in } B_{\delta_{0}}(\mathbf{1})
$$

for some fixed $\varepsilon_{0}>0$ and $\delta_{0}>0$, where $B_{\delta_{0}}(\mathbf{1})$ is the ball of radius $\delta_{0}$ centered at $\mathbf{1}=(1, \ldots, 1) \in \mathbb{R}^{n}$, which was used in the proof of boundary estimates for curvature. We achieve this by deriving a novel "global interior curvature bound" (Theorem 1.3) that also allows us to prove uniqueness of the solution for mean convex or star-shaped asymptotic boundary (Theorem 1.4 and Theorem 1.5).

An advantage in using the upper half space model of $\mathbb{H}^{n+1}$ is due to the fact that there is a remarkably simple relation between the hyperbolic $\left(\kappa_{i}\right)$ and Euclidean $\left(\kappa_{i}^{e}\right)$ principal curvatures of a hypersurface $\Sigma$ :

$$
\kappa_{i}=x_{n+1} \kappa_{i}^{e}+\nu^{n+1}, \quad 1 \leq i \leq n
$$

at $\left(x, x_{n+1}\right) \in \Sigma$, where $\nu$ is Euclidean unit normal vector to $\Sigma$ and $\nu^{n+1}=\nu \cdot e_{n+1}$.

One important consequence of (1.13) is the following result of [9].

Theorem 1.2. Let $\Sigma$ be a complete locally strictly convex $C^{2}$ hypersurface in $\mathbb{H}^{n+1}$ with compact asymptotic boundary at infinity. Then $\Sigma$ is the (vertical) graph of a function $u \in C^{2}(\Omega) \cap C^{0}(\bar{\Omega}), u>0$ in $\Omega$ and $u=0$ on $\partial \Omega$, for some domain $\Omega \subset \mathbb{R}^{n}$. Moreover, the function $u^{2}+|x|^{2}$ is strictly (Euclidean) convex.

For convenience we say $\Sigma$ has compact asymptotic boundary if $\partial \Sigma \subset$ $\partial_{\infty} \mathbb{H}^{n+1}$ is compact with respect to the Euclidean metric in $\mathbb{R}^{n}$.

According to Theorem 1.2, the asymptotic Plateau problem (1.1)(1.2) for locally strictly convex hypersurfaces reduces to the Dirichlet problem for a fully nonlinear equation of the form

$$
\begin{aligned}
G\left(D^{2} u, D u, u\right) & =\sigma, \quad u>0 \quad \text { in } \Omega \subset \mathbb{R}^{n} \\
u & =0 \text { on } \partial \Omega .
\end{aligned}
$$


In particular, the asymptotic boundary $\Gamma$ must be the boundary of some bounded domain $\Omega$ in $\mathbb{R}^{n}$. Moreover, it is also necessary to assume $0<$ $\sigma<1$ in Theorem 1.1.

The graph of a solution $u$ of equation (1.14) is locally strictly convex in $\mathbb{H}^{n+1}$ if and only if $|x|^{2}+u^{2}$ is a strictly convex function on $\Omega$. We shall call such solutions admissible. Condition (1.4) ensures that equation (1.14) is elliptic for admissible solutions, while assumption (1.5) implies that the function $G$ is concave with respect to $D^{2} u$; see [4]. By (1.3), equation (1.14) becomes uniformly elliptic on compact subdomains of $\Omega$ for admissible solutions satisfying a priori bounds in the $C^{2}$ norm and therefore allows us to apply the Evans-Krylov theorem $[6,13]$ to derive interior $C^{2, \alpha}$ and higher-order estimates.

From the above discussion we see that Theorem 1.1 is essentially optimal as far as locally strictly convex hypersurfaces are concerned. It is worthwhile to remark that we could remove condition (1.8) from Theorem 1.1. (The only conclusion that might need adjustment would be $\sqrt{1+|D u|^{2}}=\frac{1}{\sigma}$ on $\partial \Omega$.) We keep this assumption in Theorem 1.1 in order to apply results from $[\mathbf{9}, \mathbf{1 1}]$ that allow us to significantly shorten the proof. By an approximation argument we could also remove the smoothness assumption on $\Gamma=\partial \Omega$ and instead assume a uniform exterior ball condition.

The main new technical tool used in this paper is a global curvature estimate that is obtained from an interior curvature estimate. More precisely we have the following theorem.

Theorem 1.3. Suppose $f$ satisfies conditions (1.3)-(1.8) and $0<$ $\sigma<1$. Let $\Sigma$ be a smooth locally strictly convex hypersurface in $\mathbb{H}^{n+1}$ satisfying (1.1)-(1.2) with uniformly bounded principal curvatures $0<$ $\kappa_{i} \leq C=C(\Sigma)$. In the upper half-space model, let a $>0$ satisfy

$$
\nu^{n+1} \geq 2 a>0 \text { on } \Sigma
$$

and, for $\mathbf{x} \in \Sigma$, let $\kappa_{\max }(\mathbf{x})$ denote the largest principal curvature of $\Sigma$ at $\mathbf{x}$. Then for $0<b \leq \frac{a}{4}$,

$$
\sup _{\Sigma} \frac{x_{n+1}^{b} \kappa_{\max }}{\nu^{n+1}-a} \leq \frac{8}{a^{\frac{5}{2}}}\left(\sup _{\Sigma} x_{n+1}\right)^{b}
$$

In particular,

$$
\kappa_{\max } \leq 8 a^{-\frac{5}{2}} \text { on } \Sigma .
$$

The existence of such a constant $a>0$ follows from the global gradient estimates in [11]; see Corollary 2.6.

Theorem 1.1 follows from Theorem 1.3 and the existence result of [11]. To see this we approximate $\Omega$ uniformly in $C^{2}$ by smooth domains $\Omega^{\theta}$ and apply Theorem 1.2 of $[\mathbf{1 1}]$ to the curvature function $f^{\theta}:=\theta H_{n}^{\frac{1}{n}}+(1-\theta) f$ that satisfies conditions (1.3)-(1.8) as well as 
(1.12), where $H_{n}\left(\kappa_{1}, \ldots, \kappa_{n}\right)=\kappa_{1} \cdots \kappa_{n}$ corresponds to the Gauss curvature. We obtain a complete strictly locally convex hypersurface $\Sigma^{\theta}=$ $\operatorname{graph}\left(u^{\theta}(x): x \in \Omega^{\theta}\right)$ in $\mathbb{H}^{n+1}$ satisfying (1.1)-(1.2) with $f$ replaced by $f^{\theta}$. The principal curvatures of $\Sigma^{\theta}$ admit an upper bound depending on $\theta$. Moreover, $u^{\theta} \in C^{0,1}\left(\overline{\Omega^{\theta}}\right),\left(u^{\theta}\right)^{2} \in C^{\infty}\left(\Omega^{\theta}\right) \cap C^{1,1}\left(\overline{\Omega^{\theta}}\right)$ and $u^{\theta}+\left|D u^{\theta}\right| \leq C$ independent of $\theta$ by Corollary 2.6. Using Theorem 1.3, we find that $u^{\theta}\left|D^{2} u^{\theta}\right| \leq C$ where $C$ is independent of $\theta$. We can now let $\theta$ tend to zero using Evans-Krylov regularity theory to complete the proof of Theorem 1.1.

An important issue is the uniqueness of solutions to problem (1.1)(1.2). This is a complicated question even in the case of locally strictly convex hypersurfaces. From the PDE point of view, the main difficulty comes from the fact that the linearized operator of equation (1.14) may have nontrivial kernel. In this paper we are able to prove the following general uniqueness when $\Gamma$ is mean convex in $\mathbb{R}^{n}$. Throughout the rest of this paper, we assume $\Gamma=\partial \Omega \times\{0\} \subset \mathbb{R}^{n+1}$ where $\Omega$ is a bounded domain in $\mathbb{R}^{n}$. Unless otherwise stated, we also assume $\partial \Omega$ is smooth.

Theorem 1.4. Assume $\Omega$ is a $C^{2, \alpha}$ mean convex domain, i.e., the Euclidean mean curvature $\mathcal{H}_{\partial \Omega} \geq 0$. Then the solution $\Sigma$ of Theorem 1.1 is unique.

There is also uniqueness if $\partial \Omega$ is strictly (Euclidean) star-shaped about the origin. This is a well-known fact. In the following theorem we give a quantitative description in terms of the star-shapedness of the boundary; see Theorem 4.3 for more details.

Theorem 1.5. Let $\partial \Omega \in C^{2}$ be strictly (Euclidean) star-shaped about the origin. Then the unique solution given in Theorem 1.1 is strictly (Euclidean) star-shaped about the origin; i.e., $\mathbf{x} \cdot \nu>0$.

REMARK 1.6. The reader should note that in Theorem 1.3 (and Theorem 3.1 of Section 3) we are not claiming that all possible locally strictly convex solutions $\Sigma$ of (1.1)-(1.2) satisfy the global curvature bound (1.17), rather only those which are a priori known to have globally bounded principal curvatures $0<\kappa_{i} \leq C=C(\Sigma)$. We then obtain the bound (1.17) that depends only on the constant $a$ of (1.15), which in turn depends only on $\Gamma$. This is sufficient for our proof of the existence Theorem 1.1 by approximation using our earlier existence result in [11], which assumes (1.12). It is also essential in our proof of Theorems 1.4 and 1.5 which give for $C^{2, \alpha}$ mean convex domain, respectively, a $C^{2}$ strictly (Euclidean) star-shaped domain, a simpler and more direct proof of the existence of the unique locally strictly convex solution that necessarily satisfies the global curvature bound (1.17). It is entirely possible that when uniqueness fails, there exists a locally strictly convex solution $u$ of (1.14) such that $\Sigma=\operatorname{graph}(u)$ does not have uniformly bounded principal curvatures (i.e., $\sup _{\Omega} u\left|D^{2} u\right|=+\infty$ ). 
The following example may be helpful in understanding some of the subtlety of existence and regularity issues.

EXAMPLE 1.7. Take a domain $\Omega$ that can be uniformly approximated by smooth domains $\Omega^{\varepsilon}$ that satisfy a uniform exterior ball conditionfor example, an $\Omega$ with smooth boundary except at one point $P$ with a "convex conical singularity." By Theorem 1.1 we find locally strictly convex complete hypersurfaces $\Sigma^{\varepsilon}=\operatorname{graph}\left(u^{\varepsilon}\right)$ satisfying (1.1)-(1.2) with principal curvatures $\frac{1}{C^{\varepsilon}} \leq \kappa_{i}^{\varepsilon} \leq C^{\varepsilon}$. Moreover, by Corollary 2.6, $\left|u^{\varepsilon}\right|+\left|D u^{\varepsilon}\right| \leq C,\left(\nu^{\varepsilon}\right)^{n+1} \geq 2 a>0$ with $C, a$ independent of $\varepsilon$. Applying Theorem 1.3 we conclude that $\frac{1}{C} \leq \kappa_{i}^{\varepsilon} \leq C$ where $C$ is independent of $\varepsilon$. We can now let $\varepsilon$ tend to zero and obtain a smooth limiting locally strictly convex $\Sigma=\operatorname{graph}(u)$ a solution satisfying all the conditions of Theorem 1.1. This means that $u$ is globally Lipschitz and $u\left|D^{2} u\right| \leq C$ so u satisfies interior estimates similar to those satisfies by the solution of a uniformly elliptic equation.

We end with an application of Theorem 1.1 to the existence of constant curvature spacelike hypersurfaces in de Sitter space. There is a natural asymptotic Plateau problem dual to (1.1)-(1.2) for strictly spacelike hypersurfaces $[\mathbf{1 9}]$ that takes place in the steady state subspace $\mathcal{H}^{n+1} \subset d S_{n+1}$ of de Sitter space. Following Montiel [16], there is a half-space model that identifies $\mathcal{H}^{n+1}$ with $\mathbb{R}_{+}^{n+1}$ endowed with the Lorentz metric

$$
d s^{2}=\frac{1}{y_{n+1}^{2}}\left(d y^{2}-d y_{n+1}^{2}\right)
$$

It is important to note that the isometry from $\mathcal{H}^{n+1}$ to the half-space model reverses the time orientation. The dual asymptotic Plateau problem seeks to find a strictly spacelike hypersurface $S$ satisfying

$$
f(\kappa[S])=\sigma>1, \quad \partial S=\Gamma
$$

where $\kappa[S]$ denotes the principal curvatures of $S$ in the induced de Sitter metric.

If $S$ is a complete spacelike hypersurface in $\mathcal{H}^{n+1}$ with compact asymptotic boundary at infinity, then the normal vector field $N$ of $S$ is chosen to be the one pointing to the unique unbounded region in $\mathbb{R}_{+}^{n+1} \backslash S$, and the de Sitter principal curvatures of $S$ are calculated with respect to this normal vector field.

Because $S$ is strictly spacelike, we are essentially forced to take $\Gamma=$ $\partial V$ where $V \subset \mathbb{R}^{n}$ is a bounded domain and seek $S$ as the graph of a "spacelike" function $v$ :

$$
S=\left\{\left(y, y_{n+1}\right): y_{n+1}=v(y), y \in V\right\}, \quad|\nabla v|<1 \text { in } \bar{V} .
$$


In [19] we have computed the first and second fundamental forms of $S$ with respect to the induced de Sitter metric. We use

$$
X_{i}=e_{i}+v_{i} e_{n+1}, \quad N=v \nu=v \frac{v_{i} e_{i}+e_{n+1}}{w},
$$

where $w=\sqrt{1-|\nabla v|^{2}}$ and $\nu$ is the normal vector field of $\mathrm{S}$ viewed as a Minkowski space $R^{n, 1}$ graph. The first and second fundamental forms $g_{i j}$ and $h_{i j}$ are given by

$$
g_{i j}=\left\langle X_{i}, X_{j}\right\rangle_{D}=\frac{1}{v^{2}}\left(\delta_{i j}-v_{i} v_{j}\right)
$$

and

$$
h_{i j}=\left\langle\nabla_{X_{i}} X_{j}, v \nu\right\rangle_{D}=\frac{1}{v^{2} w}\left(\delta_{i j}-v_{i} v_{j}-v v_{i j}\right),
$$

respectively. Note that from (1.22), $S$ is locally strictly convex if and only if

$$
|y|^{2}-v^{2} \text { is a (Euclidean) locally strictly convex function. }
$$

There is a well-known Gauss map duality for locally strictly convex hypersurfaces in $d S_{n+1}$. For our purposes we will need a very concrete formulation of this duality [19]. Montiel $[\mathbf{1 6}]$ showed that if we use the upper half-space representation for both $\mathcal{H}^{n+1}$ and $\mathbb{H}^{n+1}$, the Gauss map $N$ corresponds to the map $L: S \rightarrow \mathbb{H}^{n+1}$ defined by

$$
L((y, v(y)))=\left(y-v(y) \nabla v(y), v(y) \sqrt{1-|\nabla v|^{2}}\right), \quad y \in V .
$$

We now identify the map $L$ in terms of a hodograph map and its associated Legendre transform. Let $p(y)=\frac{1}{2}\left(|y|^{2}-v(y)^{2}\right)$; since $p$ is strictly convex in the Euclidean sense by (1.23), its gradient map $\nabla p: V \subset \mathbb{R}^{n} \rightarrow \mathbb{R}^{n}$ is globally one to one. Define

$$
x=\nabla p(y), \quad u(x):=v(y) \sqrt{1-|\nabla v(y)|^{2}}, y \in V .
$$

Then $u$ is well defined in $\Omega:=\nabla p(V)$. The associated Legendre transform is the function $q(x)$ defined in $\Omega$ by $p(y)+q(x)=x \cdot y$ or $q(x)=$ $-p(y)+y \cdot \nabla p(y)$.

Theorem 1.8. [19]. Let $L$ be defined by (1.24) and $x$ by (1.25). Then the image of $S$ under $L$ is the hyperbolic locally strictly convex graph in $\mathbb{H}^{n+1}$

$$
\Sigma=\left\{(x, u(x)) \in \mathbb{R}_{+}^{n+1}: u \in C^{\infty}(\bar{\Omega}), u(x)>0\right\}
$$

with principal curvatures $\kappa_{i}^{*}=\kappa_{i}^{-1}$. Here, $\kappa_{1}, \ldots \kappa_{n}$ are the principal curvatures of $S$ with respect to the induced de Sitter metric. Moreover, the inverse map $L^{-1}: \Sigma \rightarrow S$

$$
L^{-1}((x, u(x)))=\left(x+u(x) D u(x), u(x) \sqrt{1+|D u(x)|^{2}}\right), \quad x \in \Omega
$$

is the dual Legendre transform and hodograph map $y=D q(x), q(x)=$ $\frac{1}{2}\left(|x|^{2}+u(x)^{2}\right)$. 
Note that when $\Sigma=\operatorname{graph}(u)$ over $\Omega$ is a strictly locally convex solution of the asymptotic Plateau problem (1.1)-(1.2) in $\mathbb{H}^{n+1}$, then its Gauss image $S=\operatorname{graph}(v)$ is a locally strictly convex spacelike graph also defined over $\Omega$ that solves the asymptotic Plateau problem $f^{*}(\kappa)=$ $\frac{1}{\sigma}>1$. We now define $f^{*}$.

Definition 1.9. Given a curvature function $f(\kappa)$ in the positive cone $K_{n}^{+}$, define the dual curvature function $f^{*}(\kappa)$ by

$$
f^{*}(\kappa):=\frac{1}{f\left(\kappa_{1}^{-1}, \ldots, \kappa_{n}^{-1}\right)}, \quad \kappa \in K_{n}^{+} .
$$

Note that $f^{*}$ may in fact be naturally defined in a cone $K \supseteq K_{n}^{+}$. For example, if $f(\kappa)=\left(\frac{H_{n}}{H_{l}}\right)^{\frac{1}{n-l}}, n>l \geq 0$ defined in $K_{n}^{+}$, then

$$
f^{*}(\kappa)=\left(H_{n-l}\right)^{\frac{1}{n-l}}
$$

is in fact defined in the standard Garding cone $K=\Gamma_{n-l}$.

Using the duality Theorem 1.8 we can transplant Theorem 1.1 to $\mathcal{H}^{n+1}$.

Theorem 1.10. Suppose $\Gamma=\partial \Omega \in C^{2}$ for a bounded domain $\Omega \subset$ $\mathbb{R}^{n}=\mathbb{R}^{n} \times\{0\}$ and $f(\kappa)$ satisfies conditions (1.3)-(1.5) and (1.7)-(1.8). Then for $\sigma>1$ there exists a complete locally strictly convex spacelike hypersurface $S$ in $\mathcal{H}^{n+1}$ satisfying $f^{*}(\kappa)=\sigma$ and $\partial S=\Gamma$ with uniformly bounded principal curvatures

$$
C^{-1} \leq \kappa_{i} \leq C \text { on } S .
$$

Moreover, $S=\operatorname{graph}(v)$ with $v \in C^{\infty}(\Omega) \cap C^{0,1}(\bar{\Omega}), v^{2} \in C^{1,1}(\bar{\Omega})$, $v\left|D^{2} v\right|+|D v| \leq C$ and

$$
\sqrt{1-|D v|^{2}}=\frac{1}{\sigma} \text { on } \partial \Omega
$$

Corollary 1.11. Under the assumptions of Theorem 1.10, there exists a complete locally strictly convex spacelike hypersurface $S$ in $\mathcal{H}^{n+1}$ satisfying

$$
\left(H_{l}\right)^{\frac{1}{l}}=\sigma>1, \quad 1 \leq l \leq n
$$

with $\partial S=\Gamma$ and having uniformly bounded principal curvatures $C^{-1} \leq$ $\kappa_{i} \leq C$ on $S$. Moreover, $S=\operatorname{graph}(v)$ with $v \in C^{\infty}(\Omega) \cap C^{0,1}(\bar{\Omega}), v^{2} \in$ $C^{1,1}(\bar{\Omega}), v\left|D^{2} v\right|+|D v| \leq C$. Further, if $l=1$ or $l=2$ (corresponding to mean curvature and normalized scalar curvature) or if $\partial \Omega$ is mean convex, we have uniqueness among convex solutions and even among all solutions (convex or not) if $\Omega$ is simply connected.

The uniqueness part of Corollary 1.11 follows from Theorem 1.6 of [11] or Theorem 1.4 and a continuous deformation argument as used in [18]. Montiel [16] proved existence for $H=\sigma>1$ (mean curvature) assuming $\partial \Omega$ is mean convex. Our result shows that for arbitrary $\Omega$ 
there is always a unique locally strictly convex solution. If $\Omega$ is mean convex the solutions constructed by Montiel must agree with the ones we construct.

An outline of the paper is as follows. In Section 2 we recall some important identities and estimates, most of them from [11], needed in the proof of our main technical result (Theorem 3.1), the "global interior curvature estimate." These identities and formulas are interesting and important in themselves and will orient the reader to our point of view. The proof of Theorem 3.1 is carried out in Section 3; Theorem 1.3 follows immediately. Theorem 1.5 and Theorem 1.4 are proved in Sections 4 and 5 , respectively; the use of Theorem 1.3 is essential in these proofs.

In the following sections, $f$ is always assumed to satisfy (1.3)-(1.8) in $K_{n}^{+}$.

Acknowledgments. This research was supported in part by the NSF and Simons Foundation.

\section{Formulas on hypersurfaces and some basic identities}

In this section we recall some basic properties of solutions of (1.1) derived in $[\mathbf{1 1}]$ that will be needed in the following sections to prove our main results.

In this paper all hypersurfaces in $\mathbb{H}^{n+1}$ we consider are assumed to be connected and orientable. If $\Sigma$ is a complete hypersurface in $\mathbb{H}^{n+1}$ with compact asymptotic boundary at infinity, then the normal vector field of $\Sigma$ is chosen to be the one pointing to the unique unbounded region in $\mathbb{R}_{+}^{n+1} \backslash \Sigma$, and the (both hyperbolic and Euclidean) principal curvatures of $\Sigma$ are calculated with respect to this normal vector field.

Let $\Sigma$ be a hypersurface in $\mathbb{H}^{n+1}$. We shall use $g$ and $\nabla$ to denote the induced hyperbolic metric and Levi-Civita connection on $\Sigma$, respectively.

Let $\mathbf{x}$ and $\nu$ be the position vector and Euclidean unit normal vector of $\Sigma$ in $\mathbb{R}^{n+1}$, respectively, and set

$$
u=\mathbf{x} \cdot \mathbf{e}, \quad \nu^{n+1}=\mathbf{e} \cdot \nu
$$

where $\mathbf{e}$ is the unit vector in the positive $x_{n+1}$ direction in $\mathbb{R}^{n+1}$, and '.' denotes the Euclidean inner product in $\mathbb{R}^{n+1}$. We refer $u$ as the height function of $\Sigma$. The hyperbolic unit normal vector is $\mathbf{n}=u \nu$.

Let $\tau_{1}, \ldots, \tau_{n}$ be local frames. The metric and second fundamental form of $\Sigma$ are, respectively, given by

$$
g_{i j}=\left\langle\tau_{i}, \tau_{j}\right\rangle, \quad h_{i j}=\left\langle D_{\tau_{i}} \tau_{j}, \mathbf{n}\right\rangle=-\left\langle D_{\tau_{i}} \mathbf{n}, \tau_{j}\right\rangle
$$

where $D$ denotes the Levi-Civita connection of $\mathbb{H}^{n+1}$. Throughout the paper we assume $\tau_{1}, \ldots, \tau_{n}$ are orthonormal so $g_{i j}=\delta_{i j}$. The principal curvatures of $\Sigma$ are the eigenvalues of the second fundamental form 
$\left\{h_{i j}\right\}$ with respect to the metric $\left\{g_{i j}\right\}$. The following formula is derived in $[\mathbf{1 1}]$ :

$$
\nabla_{i j} \frac{1}{u}=\frac{1}{u}\left(g_{i j}-\nu^{n+1} h_{i j}\right) .
$$

Let $\mathcal{S}$ be the space of $n \times n$ symmetric matrices and $\mathcal{S}^{+}=\{A \in \mathcal{S}$ : $\left.\lambda(A) \in K_{n}^{+}\right\}$, where $\lambda(A)=\left(\lambda_{1}, \ldots, \lambda_{n}\right)$ are the eigenvalues of $A$. Let $F$ be the function defined by

$$
F(A)=f(\lambda(A)), \quad A \in \mathcal{S}^{+},
$$

and denote

$$
F^{i j}(A)=\frac{\partial F}{\partial a_{i j}}(A), \quad F^{i j, k l}(A)=\frac{\partial^{2} F}{\partial a_{i j} \partial a_{k l}}(A) .
$$

We have $F^{i j}(A)=f_{i}(\lambda(A)) \delta_{i j}$ when $A$ is diagonal. Moreover,

$$
\begin{gathered}
F^{i j}(A) a_{i j}=\sum f_{i}(\lambda(A)) \lambda_{i}=F(A), \\
F^{i j}(A) a_{i k} a_{j k}=\sum f_{i}(\lambda(A)) \lambda_{i}^{2} .
\end{gathered}
$$

Equation (1.1) can therefore be rewritten locally in the form

$$
F\left(h_{i j}\right)=\sigma .
$$

Denote $F^{i j}=F^{i j}\left(h_{i j}\right), F^{i j, k l}=F^{i j, k l}\left(h_{i j}\right)$.

Lemma 2.1 ([11]). Let $\Sigma$ be a smooth hypersurface in $\mathbb{H}^{n+1}$ satisfying (1.1). Then

$$
\begin{gathered}
F^{i j} \nabla_{i j} \frac{1}{u}=-\frac{\sigma \nu^{n+1}}{u}+\frac{1}{u} \sum f_{i}, \\
F^{i j} \nabla_{i j} \frac{\nu^{n+1}}{u}=\frac{\sigma}{u}-\frac{\nu^{n+1}}{u} \sum f_{i} \kappa_{i}^{2} .
\end{gathered}
$$

Using Lemma 2.1, one derives the following important maximum principle.

Theorem $2.2([\mathbf{1 1}])$. Let $\Sigma$ be a smooth strictly locally convex hypersurface in $\mathbb{H}^{n+1}$ satisfying equation (1.1). Suppose $\Sigma$ is globally a graph: $\Sigma=\{(x, u(x)): x \in \Omega\}$ where $\Omega$ is a domain in $\mathbb{R}^{n} \equiv \partial \mathbb{H}^{n+1}$. Then

$$
F^{i j} \nabla_{i j} \frac{\sigma-\nu^{n+1}}{u} \geq \sigma(1-\sigma) \frac{\left(\sum f_{i}-1\right)}{u} \geq 0 .
$$

Upper and lower bounds on $\partial \Omega$ for $\eta:=\frac{\sigma-\nu^{n+1}}{u}$ follow from the following lemma, which is based on comparisons with equidistant sphere solutions. 
Lemma 2.3. Assume that $\partial \Sigma$ satisfies a uniform interior and/or exterior ball condition, and let $u$ denote the height function of $\Sigma$ with $u=\varepsilon$ on $\partial \Omega$. Then for $\varepsilon \geq 0$ sufficiently small,

$$
\begin{aligned}
& -\frac{\varepsilon \sqrt{1-\sigma^{2}}}{r_{2}}-\frac{\varepsilon^{2}(1+\sigma)}{r_{2}^{2}}<\nu^{n+1}-\sigma \\
& <\frac{\varepsilon \sqrt{1-\sigma^{2}}}{r_{1}}+\frac{\varepsilon^{2}(1-\sigma)}{r_{1}^{2}} \text { on } \partial \Sigma
\end{aligned}
$$

where $r_{2}$ and $r_{1}$ are the maximal radii of exterior and interior spheres to $\partial \Omega$, respectively. In particular, $\nu^{n+1} \rightarrow \sigma$ on $\partial \Sigma$ as $\varepsilon \rightarrow 0$.

\section{Corollary 2.4.}

$$
\eta:=\frac{\sigma-\nu^{n+1}}{u} \leq \sup _{\partial \Sigma} \frac{\sigma-\nu^{n+1}}{u} \text { on } \Sigma .
$$

Moreover, if $u=\epsilon>0$ on $\partial \Omega$ (satisfying a uniform exterior ball condition), then there exists $\epsilon_{0}>0$ depending only on $\partial \Omega$, such that for all $\epsilon \leq \epsilon_{0}$,

$$
\frac{\sigma-\nu^{n+1}}{u} \leq \frac{\sqrt{1-\sigma^{2}}}{r_{2}}+\frac{\varepsilon(1+\sigma)}{r_{2}^{2}} \text { on } \Sigma
$$

where $r_{2}$ is the maximal radius of exterior tangent spheres to $\partial \Omega$.

Proposition 2.5. Let $\Sigma$ be a smooth strictly locally convex graph

$$
\Sigma=\{(x, u(x)): x \in \Omega\}
$$

in $\mathbb{H}^{n+1}$ satisfying $u \geq \varepsilon$ in $\Omega, u=\varepsilon$ on $\partial \Omega$. Then at an interior maximum of $\frac{u}{\nu^{n+1}}$ we have $\frac{u}{\nu^{n+1}} \leq \max _{\Omega} u$. Hence for $\varepsilon$ small compared to $\sigma$,

$$
\nu^{n+1} \geq \frac{u}{\max _{\Omega} u} \text { in } \Omega
$$

Proof. Let $h=\frac{u}{\nu^{n+1}}=u w$ and suppose that $h$ assumes its maximum at an interior point $x_{0}$. Then at $x_{0}$,

$$
\partial_{i} h=u_{i} w+u \frac{u_{k} u_{k i}}{w}=\left(\delta_{k i}+u_{k} u_{i}+u u_{k i}\right) \frac{u_{k}}{w}=0 \quad \forall 1 \leq i \leq n .
$$

Since $\Sigma$ is strictly locally convex, this implies that $\nabla u=0$ at $x_{0}$ so the proposition follows immediately from Corollary 2.4.

q.e.d.

Combining Theorem 2.2 and Proposition 2.5 gives

Corollary 2.6. Let $\Sigma$ be a smooth strictly locally convex graph

$$
\Sigma=\{(x, u(x)): x \in \Omega\}
$$


in $\mathbb{H}^{n+1}$ satisfying $u \geq \varepsilon$ in $\Omega, u=\varepsilon$ on $\partial \Omega$. Assume that $\partial \Omega$ satisfies a uniform exterior ball condition. Then, for $\varepsilon$ sufficiently small compared to $\sigma$,

$$
\nu^{n+1} \geq 2 a:=\frac{\sigma}{1+M \max _{\Omega} u}
$$

where $M=\frac{\sqrt{1-\sigma^{2}}}{r_{2}}+\frac{\varepsilon(1+\sigma)}{r_{2}^{2}}$.

Proof. By Theorem 2.2 we have $\nu^{n+1} \geq \sigma-M u$, while by Proposition 2.5 we have $\nu^{n+1} \geq \frac{u}{\max _{\Omega} u}$. Hence if $u \leq \lambda \sigma$, we find $\nu^{n+1} \geq \sigma(1-$ $\lambda M)$, while if $u \geq \lambda \sigma$, we find $\nu^{n+1} \geq \frac{\lambda \sigma}{\max _{\Omega} u}$. Choosing $\lambda=\frac{\max _{\Omega} u}{1+M \max _{\Omega} u}$ completes the proof.

q.e.d.

\section{The global interior curvature estimate}

In this section we prove an interior curvature estimate (see Theorem 3.1 , below) for the largest principal curvature of locally strictly convex graphs with uniformly bounded principal curvatures $0<\kappa_{i} \leq C$ satisfying $f(\kappa)=\sigma$. What is remarkable is that the bound we obtain is independent of $C$ and the "cutoff" function $u^{b}$ which vanishes at $\partial \Omega$. Hence we can let $b$ tend to zero to prove the global estimate Theorem 1.3 .

Let $\Sigma$ be a smooth strictly locally convex hypersurface in $\mathbb{H}^{n+1}$ satisfying $f(\kappa)=\sigma$ with $\partial \Sigma \subset \partial_{\infty} \mathbb{H}^{n+1}$. For a fixed point $\mathbf{x}_{0} \in \Sigma$ we choose a local orthonormal frame $\tau_{1}, \ldots, \tau_{n}$ around $\mathbf{x}_{0}$ such that $h_{i j}\left(\mathbf{x}_{0}\right)=\kappa_{i} \delta_{i j}$. The calculations below are done at $\mathbf{x}_{0}$. For convenience we shall write $v_{i j}=\nabla_{i j} v, h_{i j k}=\nabla_{k} h_{i j}, h_{i j k l}=\nabla_{l k} h_{i j}=\nabla_{l} \nabla_{k} h_{i j}$, etc.

Since $\mathbb{H}^{n+1}$ has constant sectional curvature -1 , by the Codazzi and Gauss equations, we have $h_{i j k}=h_{i k j}$ and

$$
\begin{aligned}
h_{i i j j} & =h_{j j i i}+\left(h_{i i} h_{j j}-1\right)\left(h_{i i}-h_{j j}\right) \\
& =h_{j j i i}+\left(\kappa_{i} \kappa_{j}-1\right)\left(\kappa_{i}-\kappa_{j}\right) .
\end{aligned}
$$

Consequently, for each fixed $j$,

$$
F^{i i} h_{j j i i}=F^{i i} h_{i i j j}+\left(1+\kappa_{j}^{2}\right) \sum f_{i} \kappa_{i}-\kappa_{j} \sum f_{i}-\kappa_{j} \sum \kappa_{i}^{2} f_{i} .
$$

Theorem 3.1. Let $\Sigma$ be a smooth strictly locally convex graph in $\mathbb{H}^{n+1}$ with uniformly bounded principal curvatures $0<\kappa_{i} \leq C$ satisfying $f(\kappa)=\sigma, \partial_{\infty} \Sigma \subset \partial_{\infty} \mathbb{H}^{n+1}$ and

$$
\nu^{n+1} \geq 2 a>0 \text { on } \Sigma .
$$

For $\mathbf{x} \in \Sigma$ let $\kappa_{\max }(\mathbf{x})$ be the largest principal curvature of $\Sigma$ at $\mathbf{x}$. Then for $0<b \leq \frac{a}{4}$,

$$
\max _{\Sigma} u^{b} \frac{\kappa_{\max }}{\nu^{n+1}-a} \leq \frac{8}{a^{\frac{5}{2}}}\left(\sup _{\Sigma} u\right)^{b} .
$$


Proof. Let

$$
M_{0}=\sup _{\mathbf{x} \in \Sigma} u^{b} \frac{\kappa_{\max }(x)}{\nu^{n+1}-a} .
$$

Since $\kappa_{\max }(\mathbf{x}) \leq C, M_{0}>0$ is attained at an interior point $\mathbf{x}_{0} \in \Sigma$. Let $\tau_{1}, \ldots, \tau_{n}$ be a local orthonormal frame around $\mathbf{x}_{0}$ such that $h_{i j}\left(\mathbf{x}_{0}\right)=$ $\kappa_{i} \delta_{i j}$, where $\kappa_{1}, \ldots, \kappa_{n}$ are the principal curvatures of $\Sigma$ at $\mathbf{x}_{0}$. We may assume $\kappa_{1}=\kappa_{\max }\left(\mathbf{x}_{0}\right)$. Thus, at $\mathbf{x}_{0}, u^{b} \frac{h_{11}}{\nu^{n+1}-a}$ has a local maximum, and so

$$
\begin{gathered}
\frac{h_{11 i}}{h_{11}}+b \frac{u_{i}}{u}-\frac{\nabla_{i} \nu^{n+1}}{\nu^{n+1}-a}=0, \\
\frac{h_{11 i i}}{h_{11}}+b \frac{u_{i i}}{u}-\frac{\nabla_{i i} \nu^{n+1}}{\nu^{n+1}-a}-\left(b+b^{2}\right) \frac{u_{i}^{2}}{u^{2}}+2 b \frac{u_{i}}{u} \frac{\nabla_{i} \nu^{n+1}}{\nu^{n+1}-a} \leq 0 .
\end{gathered}
$$

Using (3.2), we find after differentiating the equation $F\left(h_{i j}\right)=\sigma$ twice that, at $\mathbf{x}_{0}$,

$$
F^{i i} h_{11 i i}=-F^{i j, r s} h_{i j 1} h_{r s 1}+\sigma\left(1+\kappa_{1}^{2}\right)-\kappa_{1}\left(\sum f_{i}+\sum \kappa_{i}^{2} f_{i}\right) .
$$

By Lemma 2.1 we immediately derive

$$
\begin{aligned}
F^{i j} \nabla_{i j} \nu^{n+1}= & \frac{2}{u} F^{i j} \nabla_{i} u \nabla_{j} \nu^{n+1}+\sigma\left(1+\left(\nu^{n+1}\right)^{2}\right) \\
& -\nu^{n+1}\left(\sum f_{i}+\sum f_{i} \kappa_{i}^{2}\right) \\
F^{i j} \frac{\nabla_{i j} u}{u}= & 2 \sum f_{i} \frac{u_{i}^{2}}{u^{2}}+\sigma \nu^{n+1}-\sum f_{i} .
\end{aligned}
$$

By (3.7)-(3.10) we find

$$
\begin{aligned}
0 \geq & -F^{i j, r s} h_{i j 1} h_{r s 1}+\sigma\left(1+\kappa_{1}^{2}-\frac{1+\left(\nu^{n+1}\right)^{2}}{\nu^{n+1}-a} \kappa_{1}\right) \\
& +\frac{a \kappa_{1}}{\nu^{n+1}-a}\left(\sum f_{i}+\sum \kappa_{i}^{2} f_{i}\right)-b \kappa_{1} \sum f_{i} \\
& +\left(b-b^{2}\right) \kappa_{1} \sum f_{i} \frac{u_{i}^{2}}{u^{2}}-\frac{(2-2 b) \kappa_{1}}{\nu^{n+1}-a} F^{i j} \frac{u_{i}}{u} \nabla_{j} \nu^{n+1} .
\end{aligned}
$$

Next, we use an inequality due to Andrews [3] and Gerhardt [7] that states

$$
-F^{i j, k l} h_{i j 1} h_{k l, 1} \geq \sum_{i \neq j} \frac{f_{i}-f_{j}}{\kappa_{j}-\kappa_{i}} h_{i j 1}^{2} \geq 2 \sum_{i \geq 2} \frac{f_{i}-f_{1}}{\kappa_{1}-\kappa_{i}} h_{i 11}^{2} .
$$

Recall that (see [11])

$$
\nabla_{i} \nu^{n+1}=\frac{u_{i}}{u}\left(\nu^{n+1}-\kappa_{i}\right)
$$


Thus at $\mathbf{x}_{0}$ we obtain from (3.6)

$$
h_{11 i}=\kappa_{1} \frac{u_{i}}{u}\left(\frac{\nu^{n+1}-\kappa_{i}}{\nu^{n+1}-a}-b\right) .
$$

Inserting this into (3.12), we derive

$$
-F^{i j, k l} h_{i j 1} h_{k l, 1} \geq 2 \kappa_{1}^{2} \sum_{i \geq 2} \frac{f_{i}-f_{1}}{\kappa_{1}-\kappa_{i}} \frac{u_{i}^{2}}{u^{2}}\left(\frac{\kappa_{i}-\nu^{n+1}}{\nu^{n+1}-a}+b\right)^{2} .
$$

Note that we may write

$$
\begin{aligned}
\sum f_{i}+\sum \kappa_{i}^{2} f_{i} & =\left(1-\left(\nu^{n+1}\right)^{2}\right) \sum f_{i} \\
& +\sum\left(\kappa_{i}-\nu^{n+1}\right)^{2} f_{i}+2 \sigma \nu^{n+1} .
\end{aligned}
$$

Combining (3.12), (3.14), and (3.15) gives at $\mathbf{x}_{0}$

$$
\begin{aligned}
& 0 \geq \sigma\left(1+\kappa_{1}^{2}-\frac{1+\left(\nu^{n+1}\right)^{2}}{\nu^{n+1}-a} \kappa_{1}\right)-b \kappa_{1} \sum f_{i} \\
& +\left(b-b^{2}\right) \sum f_{i} \frac{u_{i}^{2}}{u^{2}}+\frac{a \kappa_{1}}{2\left(\nu^{n+1}-a\right)}\left(\sum f_{i}+\sum \kappa_{i}^{2} f_{i}\right) \\
& +\frac{a \kappa_{1}}{2\left(\nu^{n+1}-a\right)}\left(\left(1-\left(\nu^{n+1}\right)^{2}\right) \sum f_{i}+\sum\left(\kappa_{i}-\nu^{n+1}\right)^{2} f_{i}+2 \sigma \nu^{n+1}\right) \\
& +2 \kappa_{1}^{2} \sum_{i \geq 2} \frac{f_{i}-f_{1}}{\kappa_{1}-\kappa_{i}} \frac{u_{i}^{2}}{u^{2}}\left(\frac{\kappa_{i}-\nu^{n+1}}{\nu^{n+1}-a}+b\right)^{2}+(2-2 b) \kappa_{1} \sum f_{i} \frac{u_{i}^{2}}{u^{2}} \frac{\kappa_{i}-\nu^{n+1}}{\nu^{n+1}-a} .
\end{aligned}
$$

Note that (assuming $\kappa_{1} \geq \frac{2}{a}$ and $b \leq \frac{a}{4}$ ) all the terms of (3.16) are positive except possibly the ones in the last sum involving $\left(\kappa_{i}-\nu^{n+1}\right)$, and only if $\kappa_{i}<\nu^{n+1}$.

For $\theta \in(0,1)$ to be chosen later, define

$$
\begin{aligned}
& J=\left\{i: \kappa_{i}-\nu^{n+1}<0, f_{i}<\theta^{-1} f_{1}\right\}, \\
& L=\left\{i: \kappa_{i}-\nu^{n+1}<0, f_{i} \geq \theta^{-1} f_{1}\right\} .
\end{aligned}
$$

Since $\sum u_{i}^{2} / u^{2}=|\tilde{\nabla} u|^{2}=1-\left(\nu^{n+1}\right)^{2} \leq 1, \nu^{n+1} \geq 2 a$, and $\kappa_{i} f_{i} \leq \sigma$ for each $i$, we derive

$$
\sum_{i \in J}\left(\kappa_{i}-\nu^{n+1}\right) f_{i} \frac{u_{i}^{2}}{u^{2}} \geq-\frac{f_{1}}{\theta} \geq-\frac{\sigma}{\theta \kappa_{1}}
$$

and 


$$
\begin{aligned}
2 \kappa_{1}^{2} \sum_{i \in L} \frac{f_{i}-f_{1}}{\kappa_{1}-\kappa_{i}} \frac{u_{i}^{2}}{u^{2}}\left(\frac{\kappa_{i}-\nu^{n+1}}{\nu^{n+1}-a}+b\right)^{2}+(2-2 b) \kappa_{1} \sum_{i \in L} f_{i} \frac{u_{i}^{2}}{u^{2}} \frac{\kappa_{i}-\nu^{n+1}}{\nu^{n+1}-a} \\
\geq 2(1-\theta) \kappa_{1} \sum_{i \in L} f_{i} \frac{u_{i}^{2}}{u^{2}}\left(\frac{\kappa_{i}-\nu^{n+1}}{\nu^{n+1}-a}\right)^{2}+(2+2 b-4 b \theta) \kappa_{1} \sum_{i \in L} f_{i} \frac{u_{i}^{2}}{u^{2}} \frac{\left(\kappa_{i}-\nu^{n+1}\right)}{\nu^{n+1}-a} \\
\geq \frac{2 \kappa_{1}}{\left(\nu^{n+1}-a\right)^{2}} \sum_{i \in L} f_{i} \frac{u_{i}^{2}}{u^{2}}\left(\kappa_{i}^{2}-\left(a+\nu^{n+1}\right) \kappa_{i}+a \nu^{n+1}\right) \\
\quad-\frac{2 \theta}{a} \frac{\kappa_{1}}{\nu^{n+1}-a} \sum_{i \in L} f_{i}\left(\kappa_{i}-\nu^{n+1}\right)^{2}+2 b(1-2 \theta) \kappa_{1} \sum_{i \in L} f_{i} \frac{u_{i}^{2}}{u^{2}} \frac{\left(\kappa_{i}-\nu^{n+1}\right)}{\nu^{n+1}-a} \\
\geq-\frac{6 \sigma}{a} \kappa_{1}-\frac{2 b \kappa_{1}\left(1-\left(\nu^{n+1}\right)^{2}\right)}{\nu^{n+1}-a} \sum f_{i}-\frac{2 \theta \kappa_{1}}{a\left(\nu^{n+1}-a\right)} \sum_{i \in L} f_{i}\left(\kappa_{i}-\nu^{n+1}\right)^{2} .
\end{aligned}
$$

We now fix $\theta=\frac{a^{2}}{4}$ and $0<b \leq \frac{a}{4}$. From (3.17) and (3.18) we see that the right-hand side of (3.16) at $\mathbf{x}_{0}$ is strictly greater than

$$
\sigma\left(1+\kappa_{1}^{2}-\frac{8}{a} \kappa_{1}-\frac{8}{a^{3}}\right)
$$

Then (3.19) is strictly positive if, for example, $\kappa_{1} \geq 8 a^{-\frac{3}{2}}$. Therefore, $\kappa_{1} \leq 8 a^{-\frac{3}{2}}$ at $\mathbf{x}_{0}$, completing the proof of Theorem 3.1. q.e.d.

\section{Strict Euclidean star-shapedness for convex solutions}

In this section we prove Theorem 1.5 by direct construction in Theorem 4.3 , below, of a strictly star-shaped locally strictly convex solution with boundary in the horosphere $\left\{x_{n+1}=\varepsilon\right\}$. By compactness and uniqueness we can then pass to the limit as $\varepsilon$ tends to zero. We use the continuity method by deforming from the horosphere solution $u \equiv \varepsilon$ for $\sigma=1$. Under this deformation we will show that the property of being strictly sharshaped-i.e., $\mathbf{x} \cdot \nu>0$-persists as long as a solution exists. This property is intertwined with the demonstration that the full linearized operator has trivial kernel.

Suppose $\Sigma$ is locally represented as the graph of a function $u \in C^{2}(\Omega)$, $u>0$, in a domain $\Omega \subset \mathbb{R}^{n}: \Sigma=\left\{(x, u(x)) \in \mathbb{R}^{n+1}: x \in \Omega\right\}$, oriented by the upward (Euclidean) unit normal vector field $\nu$ to $\Sigma$ :

$$
\nu=\left(\frac{-D u}{w}, \frac{1}{w}\right), w=\sqrt{1+|D u|^{2}} .
$$

The Euclidean metric and second fundamental form of $\Sigma$ are given respectively, by

$$
g_{i j}^{e}=\delta_{i j}+u_{i} u_{j}, h_{i j}^{e}=\frac{u_{i j}}{w} .
$$


According to $[\mathbf{5}]$ the Euclidean principal curvatures $\kappa^{e}[\Sigma]$ are the eigenvalues of the symmetric matrix $A^{e}[u]=\left\{a_{i j}^{e}\right\}$ :

$$
a_{i j}^{e}:=\frac{1}{w} \gamma^{i k} u_{k l} \gamma^{l j}, \quad \gamma^{i j}=\delta_{i j}-\frac{u_{i} u_{j}}{w(1+w)} .
$$

Note that the matrix $\left\{\gamma^{i j}\right\}$ is invertible and equal to the inverse square root of $\left\{g_{i j}^{e}\right\}$; i.e., $\gamma^{i k} \gamma^{k j}=\left(g^{e}\right)^{i j}$. By (1.13) the hyperbolic principal curvatures $\kappa[u]$ of $\Sigma$ are the eigenvalues of the matrix $A[u]=\left\{a_{i j}[u]\right\}$ :

$$
a_{i j}[u]:=u a_{i j}^{e}+\frac{\delta_{i j}}{w}=\frac{1}{w}\left(\delta_{i j}+u \gamma^{i k} u_{k l} \gamma^{l j}\right) .
$$

Problem (1.1)-(1.2) reduces to the Dirichlet problem for a fully nonlinear second-order equation that we shall write in the form

$$
G\left(D^{2} u, D u, u\right)=\sigma, u>0 \quad \text { in } \Omega \subset \mathbb{R}^{n}
$$

with the boundary condition

$$
u=0 \quad \text { on } \partial \Omega \text {. }
$$

The function $G$ in equation (4.3) is determined by $G\left(D^{2} u, D u, u\right)=$ $F(A[u])$ where $A[u]=\left\{a_{i j}[u]\right\}$ is given by (4.2). Let

$$
\mathcal{L}=G^{s t} \partial_{s} \partial_{t}+G^{s} \partial_{s}+G_{u}
$$

be the linearized operator of $G$ at $u$, where

$$
G^{s t}=\frac{\partial G}{\partial u_{s t}}, G^{s}=\frac{\partial G}{\partial u_{s}}, G_{u}=\frac{\partial G}{\partial u} .
$$

We shall not need the exact formula for $G^{s}$, but note that

$$
G^{s t}=\frac{u}{w} F^{i j} \gamma^{i s} \gamma^{j t}, G^{s t} u_{s t}=u G_{u}=G-\frac{1}{w} \sum F^{i i}
$$

where $F^{i j}=F^{i j}(A[u])$, etc. Under condition (1.4) equation (4.3) is elliptic for $u$ if $A[u] \in \mathcal{S}^{+}$, while (1.5) implies that $G\left(D^{2} u, D u, u\right)$ is concave with respect to $D^{2} u$.

Since $\mathbf{x} \cdot \nu=\frac{u-\sum x_{k} u_{k}}{w}$, the following lemma is important.

Lemma 4.1. We have $\mathcal{L}\left(u-\sum x_{k} u_{k}\right)=0$.

Proof. Write $\mathcal{L}=L+G_{u}$. Note that $\mathcal{L}\left(u_{k}\right)=0$ since horizontal translation is an isometry. We have

$$
\mathcal{L}\left(x_{k} u_{k}\right)=x_{k} \mathcal{L}\left(u_{k}\right)+u_{k} L\left(x_{k}\right)+2 G^{i j} \delta_{k i} u_{k j}=u_{k} G^{k}+2 G^{i j} u_{i j}=\mathcal{L} u
$$

since $G^{i j} u_{i j}=u G_{u}$.

q.e.d.

Lemma 4.2. Suppose $\mathcal{L} \phi=0$ in $\Omega, \phi=0$ on $\partial \Omega$, and there exists $v>0$ in $\bar{\Omega}$ satisfying $\mathcal{L} v=0$. Then $\phi \equiv 0$. 
Proof. Set $h=\frac{\phi}{v}$. A simple computation shows that

$$
L h+2 G^{i j} \frac{v_{i}}{v} h_{j}=0 \text { in } \Omega, h=0 \text { on } \partial \Omega .
$$

The lemma now follows by the maximum principle.

q.e.d.

Theorem 4.3. Let $\Omega$ be a strictly star-shaped $C^{2, \alpha}$ domain with respect to the origin. Suppose $f$ satisfies (1.12) in addition to (1.3)-(1.8). There exists a unique solution $u \in C^{\infty}(\bar{\Omega})$ of the Dirichlet problem

$$
G\left(D^{2} u, D u, u\right)=\sigma \text { in } \Omega, u=\varepsilon \text { on } \partial \Omega .
$$

Moreover, the hypersurface $\Sigma=\operatorname{graph}(u)$ is strictly star-shaped with respect to the origin. More precisely, there exist constants $c_{0}, \varepsilon_{0}>0$ such that for all $0<\varepsilon \leq \varepsilon_{0}$,

$$
\mathbf{x} \cdot \nu>0 \text { on } \Sigma, \quad \mathbf{x} \cdot \nu \geq c_{0} \sqrt{1-\sigma^{2}} \min _{x \in \partial \Omega} x \cdot \mathbf{N}
$$

where $\mathbf{N}$ is the exterior unit normal to $\partial \Omega$.

Proof. Consider for $0 \leq t \leq 1$, the family of Dirichlet problems

$$
\begin{aligned}
G\left(D^{2} u^{t}, D u^{t}, u^{t}\right) & =\sigma^{t}:=t \sigma+(1-t) \text { in } \Omega, \\
u^{t} & =\varepsilon \text { on } \partial \Omega .
\end{aligned}
$$

Starting from $u^{0} \equiv \varepsilon$, we shall use the continuity method to prove for any $t \in[0,1]$ that the Dirichlet problem (4.10) has a unique solution $u^{t} \in C^{\infty}(\bar{\Omega})$. Let $S$ be the set of all such $t$; we know $0 \in S$ so $S$ is not empty.

From the estimates derived in $[\mathbf{9}]$ and $[\mathbf{1 1}]$ we have

$$
\left|\left(u^{t}\right)^{2}\right|_{C^{2}(\bar{\Omega})} \leq C \forall t \in S
$$

where $C$ depends only on $\sigma$ and the exterior ball condition satisfied by $\Omega$ but is independent of $t$ and $\varepsilon$. This shows that $S$ is a closed set.

Next, let $t \in S$ and denote $w^{t}=\sqrt{1+\left|D u^{t}\right|^{2}}, \mathbf{x}^{t}=\left(x, u^{t}(x)\right)$. Then for $t>0$ sufficiently small, $w^{t} \mathbf{x}^{t} \cdot \nu^{t}=u^{t}-\sum x_{k} u_{k}^{t}>0$ and $\mathcal{L}^{t}\left(w^{t} \mathbf{x}^{t} \cdot \nu^{t}\right)=$ 0 in $\Omega$ by Lemma 4.1. By Lemma 4.2, $\mathcal{L}^{t}$ has trivial kernel. This shows that $t$ is an interior point of $S$ as long as $w^{t} \mathbf{x}^{t} \cdot \nu^{t}>0$. Since $\partial \Omega$ is strictly starshaped,

$$
\mathbf{x}^{t} \cdot \nu^{t} \geq \frac{\left|\nabla u^{t}\right|}{w^{t}}(x \cdot \mathbf{N}) \geq c_{0} \sqrt{1-\sigma^{2}} \min _{x \in \partial \Omega} x \cdot \mathbf{N} \text { on } \partial \Omega
$$

for $\varepsilon$ sufficiently small by Lemma 2.3. Hence if there is a first time $t$ such that $w^{t} \mathbf{x}^{t} \cdot \nu^{t}=0$, this occurs at an interior point, violating the maximum principle. Therefore $w^{t} \mathbf{x}^{t} \cdot \nu^{t}>0$ and so $S=[0,1]$, proving the solvability of the Dirichlet problem (4.8) and formula (4.9). q.e.d. 
Proof of Theorem 1.5. Given $f$ satisfying (1.3)-(1.8), let $f^{\theta}:=(1-$ $\theta) f+\theta H_{n}^{\frac{1}{n}}, 0<\theta<1$, which satisfies (1.12) in addition to (1.3)(1.8). By Theorem 4.3 we obtain a unique solution $u^{\theta, \varepsilon} \in C^{\infty}(\bar{\Omega})$ of the approximate problem $f^{\theta}\left(\kappa\left[u^{\theta}\right]\right)=\sigma$ with $u^{\theta, \varepsilon}=\varepsilon$ on $\partial \Omega$. Moreover, by

$$
\left|\left(u^{\theta, \varepsilon}\right)^{2}\right|_{C^{2}(\bar{\Omega})} \leq C \text { independent of } \varepsilon .
$$

Letting $\varepsilon \rightarrow 0$, we obtain a solution $u^{\theta}$ of the asymptotic problem for $f^{\theta}=\sigma$. By Theorem 1.3 the principal curvatures of $\Sigma^{\theta}=\operatorname{graph}\left(u^{\theta}\right)$ are uniformly bounded by a constant $C$ depending only on $\Omega$ and $\sigma$. Hence as $\theta \rightarrow 0$ we obtain by passing to a subsequence a smooth locally strictly convex $\Sigma$ satisfying (1.1)-(1.2) and (4.9).

q.e.d.

\section{Uniqueness for mean convex $\Omega$}

In this section we prove Theorem 1.4. We shall assume $\Omega$ is a $C^{2, \alpha}$ domain with Euclidean mean curvature $\mathcal{H}_{\partial \Omega} \geq 0$.

The main step is to show there is always a solution $\Sigma_{2}=\operatorname{graph}(u)$ of the asymptotic problem (1.1)-(1.2) in $\Omega$ with $G_{u}<0$ and moreover $u \leq v$ for any other solution $\Sigma_{1}=\operatorname{graph}(v)$. Then we show that $\Sigma_{2}$ is the unique solution. The proof we give is slightly circuitous in order to avoid delicate issues of boundary regularity caused by the degeneracy of the problem at the asymptotic boundary.

Proposition 5.1. Let $0<\sigma<1$ and $u \in C^{2}(\bar{\Omega})$ be a solution of the Dirichlet problem (4.8) for $\varepsilon>0$. Then $G_{u}<0$ in $\bar{\Omega}$. Consequently, the linearized operator $\mathcal{L}$ satisfies the maximum principle and so has trivial kernel.

Proof. Let $\Sigma=\operatorname{graph}(u)$ and $\eta \equiv \frac{\sigma-\nu^{n+1}}{u}$. Since $G_{u} \leq \eta$ by (4.7), we only need to show $\eta<0$ in $\bar{\Omega}$. According to Theorem 2.2, $\eta$ must achieve its maximum at a boundary point $0 \in \partial \Omega$. We choose coordinates so that the $x_{n}$ direction is the interior unit normal to $\partial \Omega$ at 0 where

$$
\eta_{n}=\frac{u_{n} u_{n n}}{u w^{3}}-\eta \frac{u_{n}}{u}<0 \text {, or equivalently, } \frac{u_{n n}}{w^{3}}<\eta .
$$

On the other hand, by assumptions (1.5) and (1.8),

$$
f(\kappa) \leq \sum f_{i}(\mathbf{1}) \kappa_{i}=\sum \kappa_{i} / n
$$

That is the hyperbolic mean curvature $H(\Sigma) \geq \sigma$, and therefore, equivalently,

$$
\frac{1}{w}\left(\delta_{i j}-\frac{u_{i} u_{j}}{w^{2}}\right) u_{i j} \geq n \eta .
$$

Since $\sum_{\alpha<n} u_{\alpha \alpha}=-u_{n}(n-1) \mathcal{H}_{\partial D}$, restricting (5.2) to $\partial \Omega$ implies

$$
\frac{u_{n n}}{w^{3}}-\frac{u_{n}}{w}(n-1) \mathcal{H}_{\partial \Omega} \geq n \eta \text {. }
$$


Combining (5.1) and (5.3) yields $w \eta(0)<-u_{n} \mathcal{H}_{\partial \Omega} \leq 0$. By Theorem 2.2 and the maximum principle, we obtain $\eta<0$ in $\bar{\Omega}$.

q.e.d.

Proposition 5.2. Let $\sigma \in(0,1)$. There exists a solution $u \in C^{\infty}(\Omega) \cap$ $C^{0,1}(\bar{\Omega})$ of the Dirchlet problem (4.3)-(4.4) satisfying $\left|u^{2}\right|_{C^{2}(\bar{\Omega})} \leq C$ and $G_{u}<0$ in $\Omega$.

Proof. We first assume that $f$ satisfies (1.12) in additon to (1.3)(1.8). By an existence theorem in [9], for $\varepsilon$ sufficiently small we obtain a solution $u \in C^{\infty}(\bar{\Omega})$ of the Dirichlet problem (4.8). By Proposition $5.1 G_{u}<0$ in $\bar{\Omega}$. Therefore, the linearized operator at $u$ satisfies the maximum principle and so has trivial kernel.

By the estimates in [9] and [11] we have $\left|u^{2}\right|_{C^{2}(\bar{\Omega})} \leq C$ independent of $\varepsilon$. Letting $\varepsilon$ tend to zero, we prove Proposition 5.2 assuming (1.12).

To remove the assumption (1.12) we consider $f^{\theta}$ in place of $f$ as in the proof of Theorem 1.5. From the above proof we obtain a solution $u^{\theta}$ of the asymptotic problem for $f^{\theta}=\sigma$ with $u^{\theta}=0$ on $\partial \Omega$. By Theorem 1.3 the principal curvatures of $\Sigma^{\theta}=\operatorname{graph}\left(u^{\theta}\right)$ are uniformly bounded by a constant $C$ depending only $\partial \Omega$ and $\sigma$. Let $\theta$ tend to zero, and note that the condition $G_{u} \leq 0$ is preserved in the limiting process and therefore $G_{u}<0$ in $\Omega$ by Theorem 2.2 and the strong maximum principle. We finish the proof of Proposition 5.2.

q.e.d.

Let $\hat{u}$ denote the solution of (4.3)-(4.4) constructed in Proposition 5.2. Theorem 1.4 follows from the following proposition.

Proposition 5.3. Let $v \in C^{2}(\Omega) \cap C^{0}(\bar{\Omega})$ be a solution of the Dirchlet problem (4.3)-(4.4). Then $v=\hat{u}$.

Proof. We first prove $v \geq \hat{u}$; the strict inequality holds in $\Omega$ unless $v \equiv \hat{u}$. Let $0<t \leq 1, \epsilon>0$ and $\Omega_{\epsilon}=\{x \in \Omega: d(x, \partial \Omega)>\epsilon\}$. For $\epsilon$ sufficiently small, $\partial \Omega_{\epsilon} \in C^{2, \alpha}$ and $\mathcal{H}_{\partial \Omega_{\epsilon}} \geq 0$. Applying Proposition 5.2, let $\hat{u}^{\epsilon, t} \in C^{\infty}\left(\Omega_{\epsilon}\right)$ be the solution constructed in Proposition 5.2 of the Dirichlet problem (4.3)-(4.4) in $\Omega_{\epsilon}$ with $\sigma$ replaced by $\sigma_{t}=(1-t)+t \sigma$. Note that $\sigma_{t}>\sigma$ and $v>0=\hat{u}^{\epsilon, t}$ on $\partial \Omega_{\epsilon}$ for all $0<t<1$, and $v>\hat{u}^{\epsilon, t}$ in $\Omega_{\epsilon}$ for $t$ close to zero. By the maximum principle this property must continue to hold until $t=1$. Thus as $\epsilon \rightarrow 0$ we obtain $v \geq \hat{u}$. Thus $v>\hat{u}$ in $\Omega$ or $v \equiv \hat{u}$.

Suppose now for contradiction that

$$
\max _{\Omega}(v-\hat{u})=v\left(x_{0}\right)-\hat{u}\left(x_{0}\right)>0 .
$$

Set $w^{t}:=t v+(1-t) \hat{u}$. We claim that graph $\left(w^{t}\right)$ is locally strictly convex, i.e., $\left(w^{t}\right)^{2}+\left|x-x_{0}\right|^{2}$ is strictly Euclidean convex, in a small neighborhood of $x_{0}$. At $x_{0}, \nabla v=\nabla \hat{u}$ and $D^{2} v \leq D^{2} \hat{u}$. A simple computation shows

$$
w^{t} w_{i j}^{t}-t v v_{i j}-(1-t) \hat{u} \hat{u}_{i j}=t(1-t)(v-\hat{u})\left(\hat{u}_{i j}-v_{i j}\right) \geq 0 \text { at } x_{0} .
$$


Hence, at $x_{0}$,

$w^{t} w_{i j}^{t}+w_{i}^{t} w_{j}^{t}+\delta_{i j} \geq t\left(v v_{i j}+v_{i} v_{j}+\delta_{i j}\right)+(1-t)\left(\hat{u} \hat{u}_{i j}+\hat{u}_{i} \hat{u}_{j}+\delta_{i j}\right)>0$,

and the claim follows. So $G\left(D^{2} w^{t}, D w^{t}, w^{t}\right)$ is well defined near $x_{0}$.

Note that $\frac{d}{d t} G\left(D^{2} w^{t}, D w^{t}, w^{t}\right)=\mathcal{L}^{t} w$ near $x_{0}$ where $w=v-\hat{u}$. Evaluating at $t=0$ gives

$$
\left.\frac{d}{d t} G\left(D^{2} w^{t}, D w^{t}, w^{t}\right)\left(x_{0}\right)\right|_{t=0}=\left.G^{i j}\right|_{\hat{u}} w_{i j}\left(x_{0}\right)+\left.G_{u}\right|_{\hat{u}} w\left(x_{0}\right)<0 .
$$

Hence for $t>0$ small enough, $\varphi(t):=G\left(D^{2} w^{t}, D w^{t}, w^{t}\right)\left(x_{0}\right)<\sigma$. In particular, there is a $t_{0} \in(0,1]$ such that

$$
\varphi\left(t_{0}\right)=\sigma, \varphi(t)<\sigma \text { on }\left(0, t_{0}\right) .
$$

Using the integral form of the mean value theorem, we may write

$0=\varphi\left(t_{0}\right)-\varphi(0)=\left[a^{i j} w_{i j}+b^{s} w_{s}+c(x) w\right]\left(x_{0}\right):=L w\left(x_{0}\right)+c\left(x_{0}\right) w\left(x_{0}\right)$,

where

$$
a^{i j}(x)=\left.\int_{0}^{t_{0}} G^{i j}\right|_{w^{t}} d t, b^{s}(x)=\left.\int_{0}^{t_{0}} G^{s}\right|_{w^{t}} d t, c(x)=\left.\int_{0}^{t_{0}} G_{u}\right|_{w^{t}} d t .
$$

Since graph $\left(w^{t}\right)$ is hyperbolic locally strictly convex in a small neighborhood of $x_{0}$, the operator $L=a^{i j} \frac{\partial^{2}}{\partial x_{i} \partial x_{j}}+b^{s} \frac{\partial}{\partial x_{s}}$ is elliptic in this neighborhood. Suppose for the moment that also $c\left(x_{0}\right)<0$. Then $L w\left(x_{0}\right)=-c\left(x_{0}\right) w\left(x_{0}\right)>0$, and $w$ has a strict interior maximum at $x_{0}$ contradicting the maximum principle.

We show $c\left(x_{0}\right)<0$ to complete the proof. According to (4.7),

$$
\begin{gathered}
\left.w^{t} G_{u}\right|_{w^{t}}\left(x_{0}\right) \leq \varphi(t)-\frac{1}{\sqrt{1+\left|D w^{t}\left(x_{0}\right)\right|^{2}}} \\
<\sigma-\frac{1}{\sqrt{1+\left|D \hat{u}\left(x_{0}\right)\right|^{2}}}<0 \text { on }\left(0, t_{0}\right) .
\end{gathered}
$$

Hence $c\left(x_{0}\right)=\left.\int_{0}^{t_{0}} G_{u}\right|_{w^{t}}\left(x_{0}\right) d t<0$.

q.e.d.

\section{References}

[1] M.T. Anderson, Complete minimal varieties in hyperbolic space, Invent. Math. 69 (1982), 477-494, MR 0679768, Zbl 0515.53042.

[2] M.T. Anderson, Complete minimal hypersurfaces in hyperbolic n-manifolds, Comment. Math. Helv. 58 (1983), 264-290, MR 0705537, Zbl 0549.53058.

[3] B. Andrews, Contraction of convex hypersurfaces in Euclidean space, Calc. Var. PDE 2 (1994), 151-171, MR 1385524, Zbl 0805.35048. 
[4] L. Caffarelli, L. Nirenberg \& J. Spruck, The Dirichlet problem for nonlinear second-order elliptic equations III: Functions of eigenvalues of the Hessians, Acta Math. 155 (1985), 261-301, MR 0806416, Zbl 0654.35031.

[5] L. Caffarelli, L. Nirenberg \& J. Spruck, Nonlinear second order elliptic equations IV. Star-shaped compact Weingarten hypersurfaces, Current Topics in P.D.E. Kinokunize Co., Tokyo 1986, 1-26 (Y. Ohya et al., editors), MR 1112140, Zbl 0672.35027 .

[6] L.C. Evans, Classical solutions of fully nonlinear, convex, second order elliptic equations, Comm. Pure Appl. Math. 35 (1982), 333-363, MR 0649348, Zbl 0469.35022 .

[7] C. Gerhardt, Closed Weingarten hypersurfaces in Riemannian manifolds, J. Differential Geom. 43 (1996), 612-641, MR 1412678, Zbl 0861.53058.

[8] B. Guan \& J. Spruck, Hypersurfaces of constant mean curvature in hyperbolic space with prescribed asymptotic boundary at infinity, Amer. J. Math. 122 (2000), 1039-1060, MR 1781931, Zbl 0990.53055.

[9] B. Guan, J. Spruck \& M. Szapiel, Hypersurfaces of constant curvature in Hyperbolic space I. J. Geom. Anal. 19 (2009), 772-795, MR 2538935, Zbl 1184.53066.

[10] B. Guan \& J. Spruck, Hypersurfaces of constant curvature in Hyperbolic space II, J. European Math. Soc. 12 (2010), 797-817, MR 2639319, Zbl 1197.53066.

[11] B. Guan \& J. Spruck, Convex hypersurfaces of constant curvature in Hyperbolic space. Surveys in Geometric Analysis and Relativity ALM20 (2011), 241-257, MR 2906928, Zbl 06103306.

[12] R.M. Hardt \& F.-H. Lin, Regularity at infinity for area-minimizing hypersurfaces in hyperbolic space, Invent. Math. 88 (1987), no. 1, 217-224, MR 0877013, Zbl 0633.49020 .

[13] N.V. Krylov, Boundedly nonhomogeneous elliptic and parabolic equations in a domain, Izv. Akad. Nauk. SSSR Ser. Mat. 47 (1983), 75-108 (Russian); English translation in Math. USSR Izv. 22 (1984), 67-97, MR 0688919, Zbl 0578.35024.

[14] F. Labourie, Problème de Minkowski et surfaces à courbure constante dan les variétés hyperboliques, Bull. Soc. Math. Fr. 119 (1991), 307-325, MR 1125669, Zbl 0758.53030 .

[15] F.H. Lin, On the Dirichlet problem for minimal graphs in hyperbolic space, Invent. Math. 96 (1989), 593-612, MR 0996556, Zbl 0707.35028.

[16] S. Montiel, Complete non-compact spacelike hypersurfaces of constant mean curvature in de Sitter space, J. Math. Soc. Japan 53 (2003), 915-938, MR 2003752, Zbl 1049.53044.

[17] B. Nelli \& J. Spruck, On existence and uniqueness of constant mean curvature hypersurfaces in hyperbolic space, Geometric Analysis and the Calculus of Variations, 253-266, International Press, Cambridge, MA, 1996, MR 1449411, Zbl 0936.35069.

[18] H. Rosenberg \& J. Spruck, On the existence of convex hypersurfaces of constant Gauss curvature in hyperbolic space, J. Differential Geom. 40 (1994), 379-409, MR 1293658, Zbl 0823.53047.

[19] J. Spruck \& L. Xiao, Convex spacelike hypersurfaces of constant curvature in de Sitter space, Discrete and Contin. Dyn. Syst. seriesB 17 (2012), 2225-2242, MR 2924459, Zbl 1248.53045.

[20] Y. Tonegawa, Existence and regularity of constant mean curvature hypersurfaces in hyperbolic space, Math. Z. 221 (1996), 591-615, MR 1385170, Zbl 0874.53050. 
Department of Mathematics Ohio State University Columbus, OH 43210

E-mail address: guan@math.osu.edu

Department of Mathematics JOHNS HOPKINS UNIVERSITY BALTimore, MD 21218

E-mail address: js@math.jhu.edu

Department of Mathematics JOHNS HOPKINS UNIVERSITY BALTimore, MD 21218

E-mail address: lxiao@math.jhu.edu 